\title{
Study of centroiding algorithms to optimize Shack-Hartmann WFS in the context of ELTs
}

\author{
Sandrine Thomas ${ }^{1, a}$, Olivier Lardiere ${ }^{2}$, Donald Gavel ${ }^{1}$, and Rodolphe Conan ${ }^{2}$ \\ 1 UCO/Lick Observatories, University of Santa Cruz, 1156 High Street, 95064 Santa Cruz, CA, USA \\ 2 The University of British Columbia 2329 West Mall Vancouver, BC Canada V6T 1Z4
}

\begin{abstract}
Along with the expected ELTs comes the diversification of Adaptive Optics (AO) systems. Most AO are beginning to make extensive use of laser guide stars (LGS), to increase sky coverage and produce a bright beacon in order to reduce wavefront measurement errors and improve performance. However, because of the larger size of the next generation of telescopes, the elongation seen in a Shack-Hartmann Wavefront Sensor is larger, i.e up to 7" for a 30m telescope. With such an elongation, both photon and read noise will increase and distribution variations of sodium atoms in the sodium layer start to matter. In this paper, we conduct ShackHartmann simulations at the sub-aperture level made with real sodium profiles taken at Lick Observatory. We will compare focus on two methods of centroiders: the matched filter and the correlation. We then compare results with data taken form a real bench at Uvic in the particular case of NFIRAOS AO system for TMT. The goal is to better understand the impact of such variations on the final error budget for the WFS.
\end{abstract}

\section{Introduction}

The next generation of adaptive optics (AO) is synonym of large telescopes and extensive use of laser guide stars (LGS). Laser guide stars have been used successfully on 4 to $10 \mathrm{~m}$ class telescopes for a few years now [1]. Implementing them on larger telescopes becomes a challenge, especially for ShackHartmann (SH) wavefront sensing. Indeed, a SH wavefront sensor samples the wavefront coming from a reference star in subapertures using lenslet array and measures local tilts of the wavefront from the displacement of each image points made with each lenslet. Because of the thickness of the sodium layer, using a LGS becomes problematic on larger telescopes. Viewed from the outer edge subapertures of a Shack-Hartmann, the spots are strongly elongated. Moreover, the distribution of sodium atoms varies with time creating a variation in the average altitude of the layer. Firstly, photons are spread over more pixels, reducing the SNR for a given laser power. Furthermore, due to the elongation, the variations of the atoms of sodium distribution will be resolved at the outer edges, leading to erroneous wavefront measurements, being mostly defocus. The main sources of error in equation are [2], [3],[4]:

$$
E_{\text {total }}=E_{\text {noise }}+E_{\text {Lin }}+E_{\text {Sodium }}+E_{\text {resp }}+E_{\text {Diff }}+E_{\text {atm }}
$$

$E_{\text {noise }}$ includes the CCD noise sources (readout noise and dark current), photon noise and the background noise due to Rayleigh scattering of the projected laser light. $E_{\mathrm{Lin}}$ is due to non-linearity because of under-sampling and truncation of the LGS spot image. They have also been studied in [3] in the context of Gaussian spot. $E_{\text {sodium }}$ is the error due to the structure and time variability in the LGS images due to variability of the sodium layer, $E_{\text {resp }}$ is due to the response coefficient not equal to unity, $E_{\text {Diff }}$ the error due to the diffraction spikes in each SHWFS subaperture, and, $E_{\text {atm }}$ is the error due to the atmosphere considering only speckle. We will not focus on this term in this paper.

The main goal of this paper is to study the impact of the sodium variations on the wavefront measurements and compare $E_{\text {sodium }}$ to $E_{\text {noise }}$. The field of view of each subaperture considered in this paper will be large enough to neglect the non-linearity effects. The geometry used here is the normal cartesian CCD. In this paper, we will only talk about the case of the central launch, leading to symmetric spots, to quantify the impact of the sodium variations on the wavefront error.

\footnotetext{
a e-mail: author@cnrs.fr
} 
Section 2 shows the method used to simulate the images obtained on the WFS camera. In section 3 we describe the case of the TMT with no noise to estimate the error due to the sodium layer itself. To verify our simulations, we compared them to laboratory experiments from the University of Victoria in section 4 . Finally we compared the error due to the sodium layer variations against the error due to photon and readout noise.

\section{Method}

In order to study the impact of the sodium variations on the centroid calculation, we simulated a full Shack-Hartmann WFS using the profiles presented in [5]. We dispose of a total of 97 images (giving us 30000 synthetic profiles) taken during 6 nights from November 2005 until March 2008. Each image represents a drift scan of about 300ms (cf. Fig. 1-far left). From those images, we take an horizontal line and put this line into a 2D matrix with the appropriate elongation set by the position of the subaperture considered with respect to the center of the CCD. We then convolve this image with the diffraction pattern cause by one subaperture and the Gaussian approximation of a seeing spot (see Fig.1). We repeat this calculation for all subapertures. As a reminder, we are considering a laser launch from the center of the pupil. The resulting image is shown on Fig.1-right. On this image one can clearly see the asymmetry of the spot.

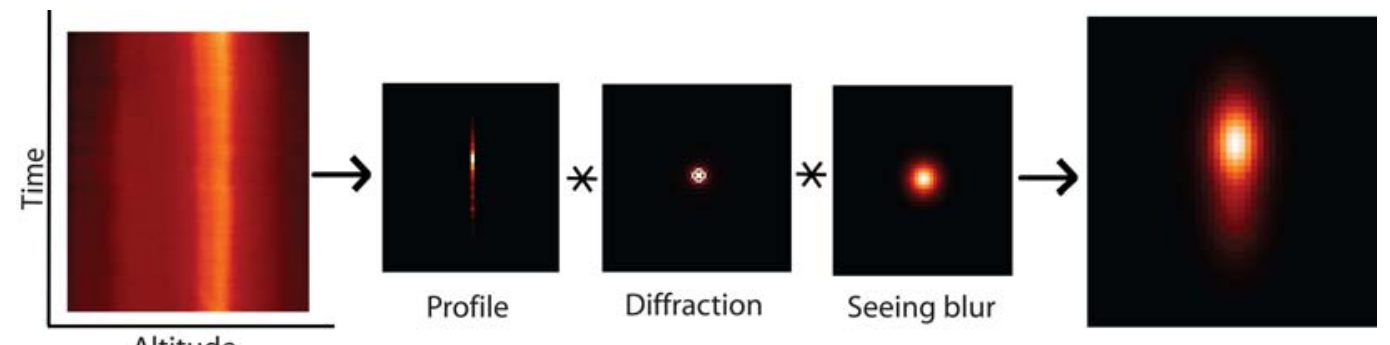

Altitude

Fig. 1. Scheme of the method of simulating an elongated spot at the outer edge subaperture.

Once the images are simulated, we use the algorithms described in [3]. However, for sake of clarity, we present results for three main methods only: thresholded center of gravity (TCoG), the correlation tracking (CORR) [6] and the Weighted Least Squares algorithm (WLS also called matched filtering) proposed by [7]. The threshold value for the normal Thresholding is $10 \%$ of the maximum [8] and the threshold for the peak finder in the correlation method is $1 \%$ at high SNR and $60 \%$ at low SNR. Another useful algorithm not described in this paper is the weighted centroid [9], [3], [10]. The next step is to reconstruct the wavefront using a Fourier Transform reconstructor [11]. Finally we look at the Zernike decomposition to analyze which aberrations are introduced and how significant they are.

One important and somewhat fastidious aspect of the process is the choice of the references, both for the spot position itself and for the correlation and matched filtering algorithms. As mentioned above, the sodium layer average altitude varies significantly leading to shift of the centroid. To estimate this shift, one can use simultaneous high resolution measurements of the sodium layer or use previous SHWFS images. To be consistent with the TMT study and Uvic configuration, we will use an average over about $16 \mathrm{~ms}$ as a reference. In an other paper, we will study in more detail the impact of the choice of the references.

We conducted this study in the particular case of the Thirty Meter Telescope (TMT) [12], with comparison with laboratory experiments from UVIC. Table 1 gathers the parameters used for the simulations. For the specific case of the wavefront sensor, we used a sampling of 2 pixels per FWHM. Also, to match the configuration of the experiments at Uvic [13], we did not simulate the polar coordinate CCD [14] but a cartesian coordinates CCD. We will focus on the effect of the sodium layer temporal and spatial variations in the context of the Polar CCD in a follow-up paper. 
Table 1. Ranges of parameters for TMT study relevant for this paper.

\begin{tabular}{ll}
\hline Parameter & Values \\
\hline Number of photons & $N_{p h}$ from $2 \times 10^{2}$ to $10^{4}$ photons \\
Readout noise & $N_{r}$ from 1 to $5 \mathrm{e}^{-}$ \\
Sodium (Na) layer thickness & from $10 \mathrm{~km}$ to $20 \mathrm{~km}$ \\
Operating wavelength & $589 \mathrm{~nm}$ \\
Spot size (non-elongated) & 1.19 "(typical seeing, no uplink correction) \\
Telescope diameter & $30 \mathrm{~m}$ \\
Number of subapertures & $60 \times 60$ \\
Subaperture field of view & 9.5 " (or 16 by 16 pixels) \\
Sampling & 2 pixels per FWHM \\
\hline
\end{tabular}

To be more complete, we simulated the shack-Hartmann behavior for different profiles, quiet and turbulent. We call a quiet profile, a profile that does not have a lot of distribution changes over a range of $300 \mathrm{sec}$. Fig. 2-left from October 10th 2006 and Fig. 2-center from February 9th 2006 show examples of such quiet profiles, where the abundance was very stable over $300 \mathrm{~ms}$ and concentrated in a well defined altitude. A more turbulent night would be similar to Fig. 2-right, where the abundance of the sodium atoms varies noticeably over the $300 \mathrm{sec}$.
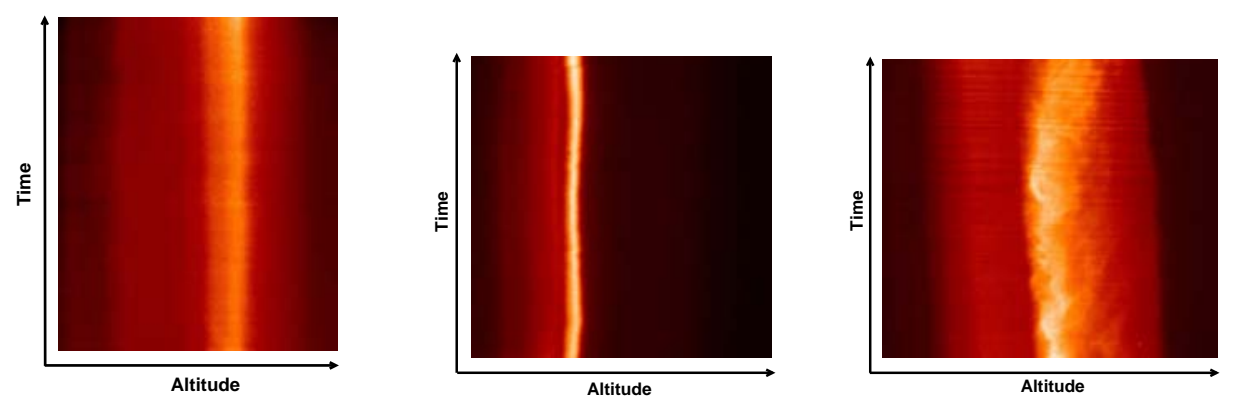

Fig. 2. Profiles used in the simulations. The two profiles on the left are examples of "quiet" profiles and on the right an example of a turbulent one. The total time of each drift scan is of the order of $300 \mathrm{~ms}$. The altitude sampling is $26 \mathrm{~m}$ per pixels.

\section{No noise configuration in the case of TMT}

We first isolate the error due to the fluctuations of the sodium layer by choosing no readout nor photon noise. The other parameters are set to the values presented in table 1. As mentioned before, we use an average over a few template as a reference both for the reference spot and the reference shape for correlation and match filter method.

Fig. 3 shows the Zernike coefficients rms values for the three profiles presented in the previous section. As expected, the main aberration is defocus (Z4) and is equal to about $60 \mathrm{~nm}$ rms. In addition we find radially symmetric aberrations (spherical (Z11), second order Spherical (Z22), quadrature trefoil aberrations Z14 and Z26). The rms value of those aberrations spread from 1 to $8 \mathrm{~nm}$. Also, we notice that all algorithms give very similar results to less than $1 \mathrm{~nm}$ rms. Also, the resulting Zernike coefficient value depend on the centroiding method and the type of aberration. Depending on the night, the main aberrations can vary as much as $2 \mathrm{~nm}$ rms. Aberration Z14 is very sensitive to both the night 

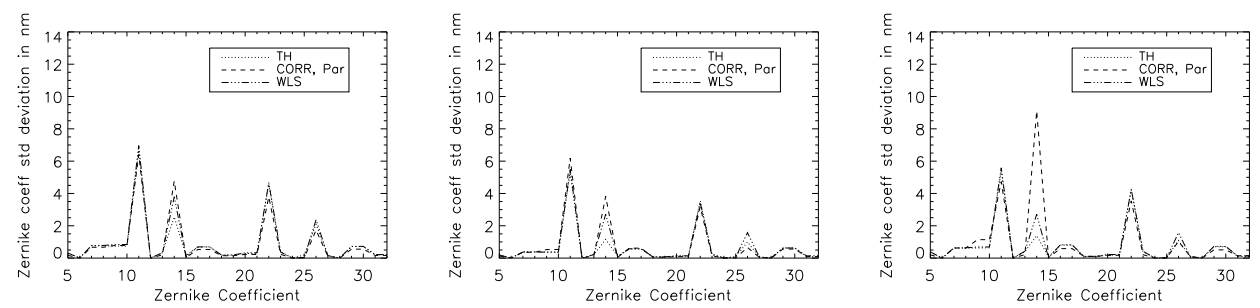

Fig. 3. From left to right. Zernike coefficient of the wavefront for October 10th 2006. Zernike coefficient of the wavefront for the "quiet" profile of February 9th 2006. Zernike coefficient of the wavefront for the "more turbulent" profile of February 9th 2006.

and the centroiding algorithm. However, we can not explain this sensitivity yet and more study will be done before drawing any conclusions.

Table 2. Defocus evolution

\begin{tabular}{lcccccc}
\hline Night & CoG & thresholding & WCoG & Corr & Corr Par & WLS \\
\hline Oct 10th 2006, 178 & 40.3 & 42.9 & 43.8 & 40.6 & 36.8 & 43.7 \\
Feb 9th 2006, 50 & 31.9 & 35.0 & 37.2 & 32.3 & 32.4 & 36.3 \\
Feb 9th 2006, 66 & 63.5 & 68.8 & 70.1 & 64.1 & 54.1 & 70.3 \\
\hline
\end{tabular}

In table 2, we present the evolution of defocus for the three nights and the different centroiding algorithms. The best performance so far in our study give a defocus of $30 \mathrm{~nm}$, which is within the error budget allocated for the TMT. Moreover it is planned in TMT to correct for the defocus, potentially by measuring it with an extra telescope. The other aberrations give a few nm of wavefront error as well, still negligible. Especially compared to the error introduced by noise as we will present in the next section.

\section{Noise configuration}

\section{Comparison to Uvic bench.}

The next step is to add photon and readout noise to evaluate the relative importance of the error resulting from the sodium variations. To confirm our simulations, we first compared them to results obtained with the optical bench experiment located at the University of Victoria, Canada [13]. The configuration differs from the TMT only in the number of subapertures (32x32). Since the telescope aperture is kept to $30 \mathrm{~m}$, this leads to subaperture field of view twice as big as in the previous section. The comparisons were conducted both at low SNR (18) and high SNR (140) corresponding to the following combinations of photon and readout noise $\{900,5\}$ and $\{20000,1\}$. Also, the number of pixels per subaperture used in the simulations is 16 by 16 pixels while in the experiments we used 15 by 15 . For this paper, comparisons have been done only in the case of one profile (Oct 10th 2006).

For high SNR, the simulations show that changes in the sodium abundance lead to the same aberrations as the case without noise described in section 3. However, the noise floor due to photon and readout noise is now $0.5 \mathrm{~nm}$ rms. The results from UVIC's laboratory show the same aberrations with slightly lower amplitude (about 1-2 nm), which might be due to unwanted differences in SNR.

Compared to the case of high SNR, the low SNR (18) lead to more asymmetric types of aberrations such as Z6, Z9 and Z20-Z21 (Fig. 4), both for simulations and experiments. The defocus is similar to the case of high SNR, i.e. $60 \mathrm{~nm}$, which is unsurprising because it predominates the errors due to photon and readout noise. The other aberrations however are on the verge to be limited by noise, meaning that for even lower SNRs, the error due to the variations of atoms abundance in the sodium 
layer will be negligible. Also, the correlation with parabolic fit of the correlation function gives the best results both for simulations and experimentations (cf. Fig. 4, dashed line on the left graph and red curve on the right graph). Finally the noise floor now depends on the Zernike aberration and varies from $4 \mathrm{~nm}$ for $\mathrm{Z} 5$ to about $2 \mathrm{~nm}$ for Z28. Future work should include more thorough analysis and some kolmogorov simulations.

The results from the laboratory at Uvic are again very similar. The graph on Fig. 4 shows the apparition of the same non-symmetric aberrations, Z6, Z9, Z20-Z21, with the same amplitude within $1 \mathrm{~nm}$. The noise floor also is at the same level as the simulations within $1 \mathrm{~nm}$. The lower level of noise agrees with the hypothesis that the mismatch is due to a difference in SNR.
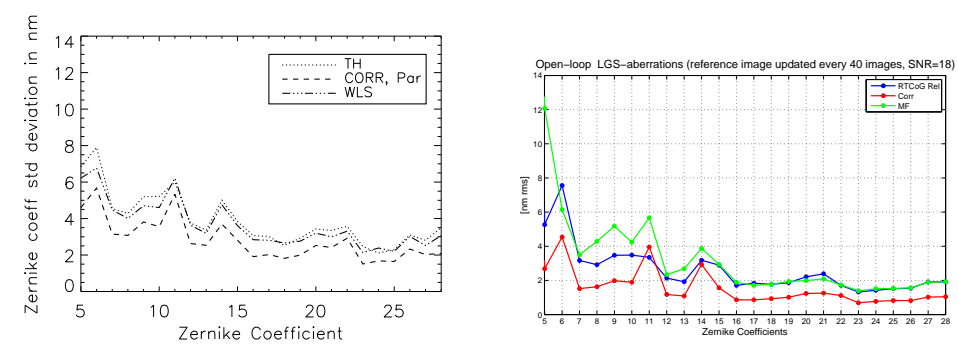

Fig. 4. Results obtained in the case of a SNR of 18 for October 10th 2006. Left graph shows the results from the simulations and the right graph from the experiments at UVIC.

Simulations and experiments are sufficiently in agreement to now switch to a more realistic configuration for the TMT, i.e. using 60x60 subaperture and in presence of readout and photon noise.

\section{Case of the TMT.}

Fig. 5 shows the results for the three different profiles presented in section 3. Results do not change
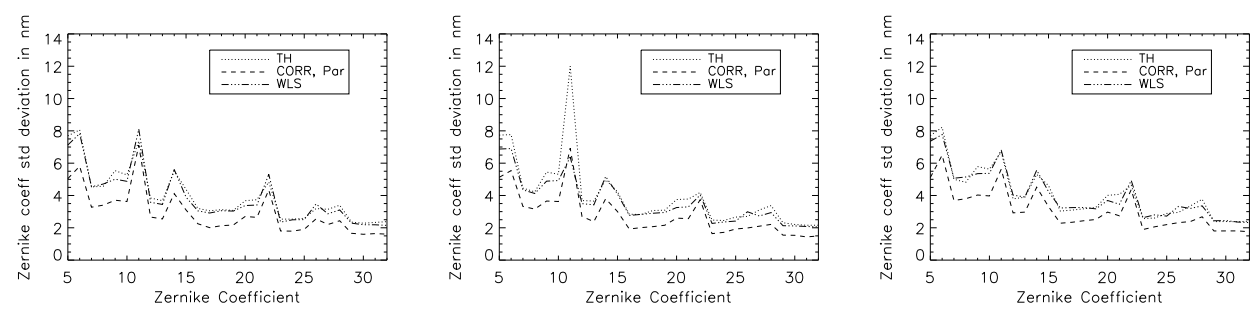

Fig. 5. From left to right. Zernike coefficient of the wavefront for October 10th 2006. Zernike coefficient of the wavefront for the "quiet" profile of February 9th 2006. Zernike coefficient of the wavefront for the "more turbulent" profile of February 9th 2006.

drastically when increasing the number of subapertures and therefore decreasing the field of view of each them. We indeed find the same aberrations with the same amplitude within less than $1 \mathrm{~nm}$ (Fig. 5-left). Surprisingly, the aberration amplitudes found did not vary by more than a few $\mathrm{nm}$ for the three different distribution variations. One explanation is that we might still not be resolving those variations. Also, for the particular profiles we considered, the atoms of sodium might be spreading over a thicker layer without significantly changing the position of the centroid. At this point, more simulations are needed to draw more significant conclusions. 


\section{Conclusions}

This paper shows some preliminary results of simulations of an LGS SHWFS using real sodium layer profiles to simulated the elongated spot. We first demonstrated that using identical profiles, the expected aberrations introduced by the variations in the sodium layer were identical within $2 \mathrm{~nm}$ for both simulations and experimental data. Moreover, the aberrations are either radially symmetric or in agreement with the geometry of the SH image. The main aberration is defocus and is of the order of 60nm. This can be easily corrected by deformable mirrors (DMs) in the system, since it is a small fraction of most of nowadays DM's total strokes. Follow up work will include more simulations with other profiles to detect a trend in aberrations. We will also study the case of the polar CCD [14].

Acknowledgements The authors would like to gratefully acknowledge the Gordon and Betty Moore Foundation for postdoctoral partial support of Dr. Thomas via the Laboratory for Adaptive Optics at UC Santa Cruz.

\section{References}

1. Foy, R., Labeyrie, A., "Feasibility of adaptive telescope with laser probe", A\&A 152, (1985), L29L31

2. Clare R. M., van Dam M. A., Bouchez A. H., "Modeling low order aberrations in laser guide star adaptive optics systems", Optics Express, 15, (2007), 4711-4725

3. Thomas, S., J., Fusco, T., Tokovinin, A., Nicolle, N., Michau, V. and Rousset, G., " Comparison of centroid computation algorithms in a Shack-Hartmann sensor", MNRAS 371, (2006), 323-336

4. Thomas, S. J., Adkins, S., Gavel, D., Fusco,T. and Michau,V., "Study of optimal wavefront sensing with elongated laser guide stars", MNRAS 387, (2008), 173-187

5. Thomas, S. J., Gavel, D., Adkins,S., Kibrick R., "Analysis of on-sky sodium profile data”, App. Optics, (2009), submitted

6. Michau V., Conan J.-M., Fusco T., Nicolle M., Robert C., Velluet M.-T., Piganeau E., SPIE 6303, (2006), 63030B

7. Gilles, L. and Ellerbroek, B., "Shack-Hartmann wavefront sensing with elongated sodium laser beacons: centroiding versus matched filtering", Applied Optics IP 45, (2006), 6568-6576

8. Lardière, O., Conan, R. , Bradley, C., Jackson, K., Hampton, P., "Radial thresholding to mitigate laser guide star aberrations on centre-of-gravity-based Shack-Hartmann wavefront sensors", MNRAS 398, (2009), 1461-1467

9. Nicolle, M., Fusco, F., Rousset, G., Michau, V., "Improvement of Shack-Hartmann wave-front sensor measurement for extreme adaptive optics", Optics Letters 29, 2743-2745 (2004).

10. Schreiber, L. , Foppiani, I., Robert, C., Diolaiti, E., Conan, J.-M., Lombini, M., "Laser guide stars for extremely large telescopes: efficient Shack-Hartmann wavefront sensor design using the weighted centre-of-gravity algorithm", MNRAS 396, (2009), 1513-1521

11. Poyneer, L. A.; Dillon, D., Thomas, S., Macintosh, B. A., "Laboratory demonstration of accurate and efficient nanometer-level wavefront control for extreme adaptive optics",Applied Optics 47, (2008), 1317-1326

12. Herriot, G., Hickson, P., Ellerboek, B. L., Andersen, D. A., Davidge, T., Erickson, D.A., Powell, I. P., Clare, R., Gilles, L., Boyer, C., Smith, M., Saddlemyer, L. and Véran, J.-P., " NFIRAOS: TMT narrow field near-infrared facility adaptive optics", Proc. SPIE 6272, (2006), 62720

13. Conan, R., Lardière, O., Herriot, G., Bradley, C., Jackson, K., "Experimental assessment of the matched filter for laser guide star wavefront sensing", Applied Optics 48, p.1198

14. Adkins, S., Azucena, O. and Nelson, J., "The design and optimization of detectors for adaptive optics wavefront sensing", Proc. SPIE 6272, (2006), 62721E 